\title{
The dynamics of heroin use; implications for intervention
}

\author{
T Millar, N Craine, T Carnwath, M Donmall
}

\begin{abstract}
Study objective-To use a readily available dataset to detect periods of epidemic change and to examine the progression of heroin epidemics in different geographical areas. To consider the implications of epidemic change for strategies to tackle drug misuse.

Design-Comparison of trends in new treatment demand, observed incidence, and age specific population rates for treated heroin users in two geographical areas.

Participants-Heroin users recorded to have sought treatment.

Main results-The areas studied seem to show differences with respect to trends in new treatment demand, incidence of heroin use and distribution of age specific population rates; indicating that they may be at different epidemic stages.

Conclusions-These analyses show how areas may differ with respect to epidemic progression of heroin use. It is essential that government strategies, and local responses to these, should be cognisant of these dynamics.
\end{abstract}

(F Epidemiol Community Health 2001;55:930-933)

The British government's 10 year strategy for drug misuse emphasises the importance of research in assessing the efficacy of drug misuse interventions. ${ }^{1}$ This strategy sets targets for interventions and proposes performance indicators including: (1) increased participation of problem drug users in drug treatment programmes and (2) a reduction in the number of drug related deaths. The emphasis on such indicators is both welcome and important. However, if these indicators are to have cross sectional or longitudinal comparative value, then it is imperative that the underlying dynamic of a heroin epidemic in the geographical region is carefully considered. The state of an epidemic before a new intervention strategy will have important and complex interactions with the proposed performance indicators.

Epidemics of heroin use have been previously reported ${ }^{2-6}$ and a variety of researchbased methods have been applied to estimating the prevalence of hidden drug misuse. ${ }^{7}$ However, in addition to quantifying point prevalence, policy makers require information about trends over time. ${ }^{8}$ Here we show how a secondary surveillance dataset, which is readily and routinely available to the strategic groups responsible for implementing national policy at the level of local government, can be examined to detect periods of epidemic change. We present data from two urban areas less than 10 miles apart in Greater Manchester, north west England. Our primary aim was to determine whether this dataset might provide an indicator of past trends in the relative incidence of heroin use. This may provide early warning of increases in the prevalence of heroin use and of changes in the future composition of the target population.

\section{Methods}

The analysis utilised data on heroin users seeking treatment and reported to the University of Manchester Drug Misuse Database (DMD) between 1988 and 1999. This system has been shown to provide an accurate measure of drug problems presenting to community drug teams (CDTs) in north west England ${ }^{9}$ and is an established treatment surveillance mechanism in this area. This data source is available in the majority of UK regions.

We have determined the number of heroin users in each area making their first treatment demand each half year between 1988 and 1997. General practitioner (GP) and CDT reports were analysed independently. First treatment demand is defined here as the first occasion on which a person was reported to DMD to have sought treatment.

Temporal changes in lag between first use and first treatment demand might potentially confound this analysis, hence we have also examined year of onset of heroin use, between 1986 and 1992, for cases seeking treatment between 1986 and 1999. Year of onset was derived from year of birth and age (in years) at onset. This straightforward approach eliminates the effect of changes in lag by assigning cases to the year in which they started to use, rather than that in which they sought treatment. This provides an indication of relative changes in observed incidence rates.

A variety of factors may influence changes in treatment demand, irrespective of changes in community prevalence. Hence the number of heroin users making new treatment demands may not have a direct relation to the number starting use in the community. Further analyses compared trends in age specific population rates for all users seeking treatment between 1988 and 1997, to determine whether changes in the age composition of the treated population might provide additional corroboration of observed trends in treatment demand and relative incidence.

Trends in age of first use of heroin, lag between first use and first treatment demand, and the route of heroin use among those newly presenting for treatment were also examined. 


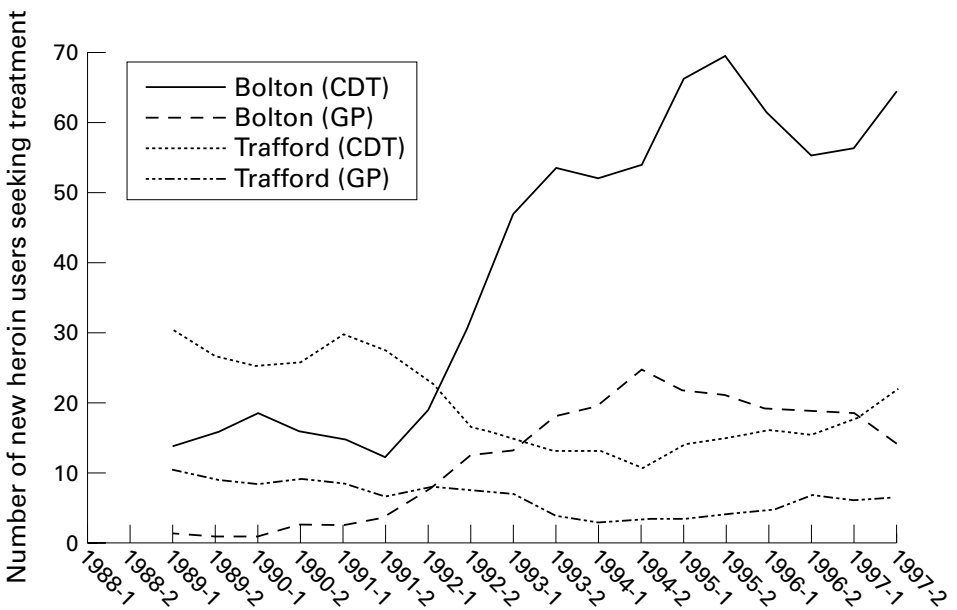

Figure 1 Three point moving average of six month totals of general practitioner and community drug team reports of new treatment demands by heroin users: 1988 to 1997.

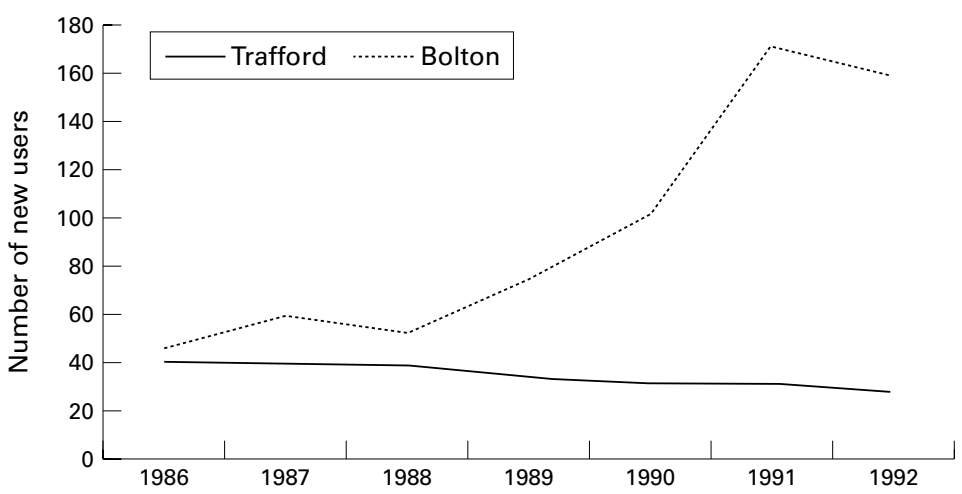

Figure 2 Relative trends in back calculated observed incidence: number of new heroin users by year of onset: 1986 to 1992.

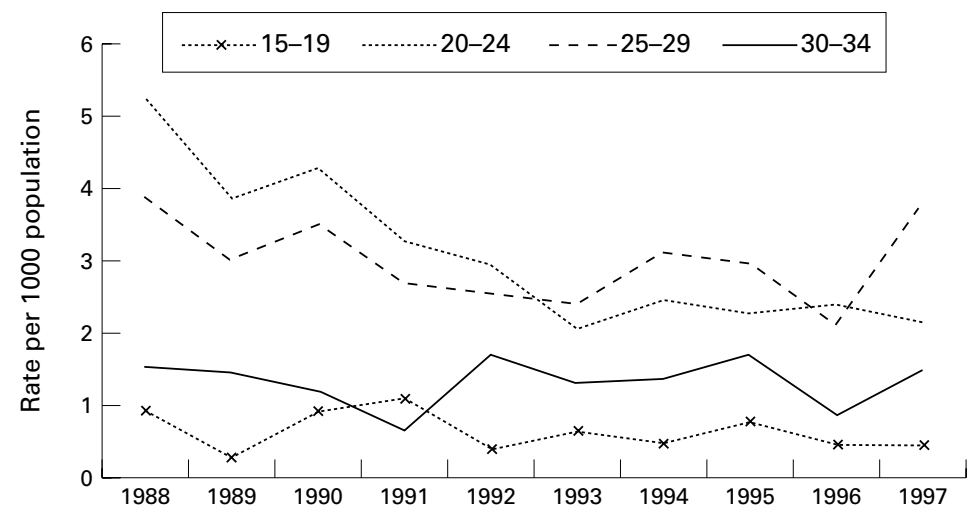

Figure 3 Trafford. Trends in age specific population rates: number of heroin users seeking treatment per 1000 population by year: 1988 to 1998.

\section{Results}

Analysis indicates distinct trends in the extent of new treatment demands (fig 1). New demands in one area (Trafford) declined between 1988 and 1992 and remained relatively stable thereafter. In the other (Bolton), they increased between 1988 and 1995, and declined thereafter. Within each area, similar patterns were seen in both GP and CDT data. Because these findings are based on first treatment demand, which post-dates first heroin use, this may indicate an earlier change in the population starting to use heroin.

Analysis by year of onset of use (fig 2) indicates that the number of persons known to have started heroin use in Bolton increased each year between 1988 and 1991, from 52 known new cases to 171. In Trafford the number of known new cases declined each year, from 40 in 1986 to 27 in 1992.

In Trafford, analysis of trends in age specific annual population rates (fig 3 ) indicates a pattern consistent with stable or declining incidence. Rates for treated heroin users aged 20-24 years declined from 5.3 to 2.1 per thousand, falling below those for 25-29 year olds. Rates for 25-29 and 30-34 year olds fluctuated in a range between $2.1-3.9$ and $0.7-1.7$ per thousand, respectively. By contrast, analysis for Bolton (fig 4) indicates a shift in population rates that is consistent with an epidemic phase. Between 1988 and 1991, rates for all age groups clustered within a relatively narrow band. Rates increased from 1991, but the increase was more rapid and of proportionally greater magnitude among younger age groups. Rates for 15-19 year olds increased from 0.3 to 7.1 per thousand between 1991 and 1995, but declined thereafter. Those for 20-24 year olds increased from 1.4 to 11.8 per thousand between 1991 and 1997. For 25-29 year olds they increased from 1.7 to 4.8 per thousand, peaking in 1995. For 30-34 year olds they increased from 0.5 to 2.7 per thousand.

Analysis (not shown) of route of administration of heroin among those newly presenting for treatment in Trafford indicates no clear trend. In most years, although these fluctuate, similar numbers and proportions of injecting and non-injecting users were reported. Analysis for Bolton seems to indicate the emergence of non-injecting as a more common mode of administration. Starting from a very low baseline, non-injecting became the modal route of administration by the latter part of the period studied, but declined thereafter. Although the emergence of non-injecting use among treatment cohorts may be indicative of an epidemic phase characterised by smoking heroin, sample sizes for early years are too small to permit more meaningful analysis.

Examination of lag between onset of use and first treatment demand indicates a reduction in lag for Bolton. Lag in Trafford changed little over the period of study. Median age of first heroin use fluctuated between 18 and 20 years in Trafford and 19 and 20 years in Bolton but showed no clear upward or downward trend in either area.

\section{Discussion}

It is clear that the stage of an epidemic must influence the composition and future size of the using population in the area affected. In view of this, strategies to tackle drug misuse must consider underlying epidemic dynamics, as must long term evaluation of these strategies. Here we have presented data from a source that is readily available to those responsible for strategic planning and analyses that can be carried out directly, without the need for additional research. Analyses for one area suggest a recent epidemic and, for the other, relatively stable incidence of heroin use. 


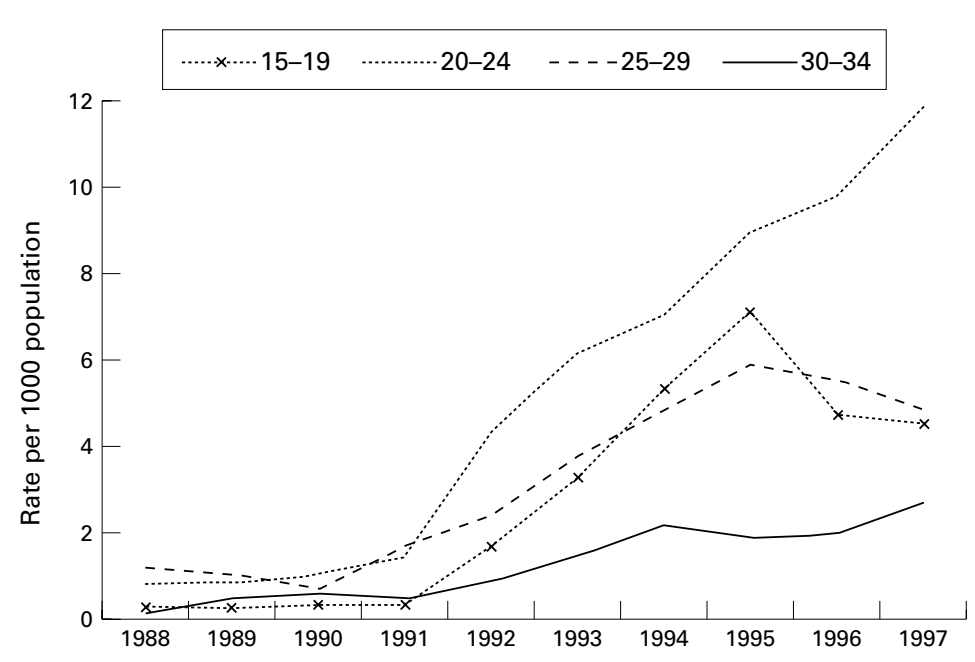

Figure 4 Bolton. Trends in age specific population rates: number of heroin users seeking treatment per 1000 population by year: 1988 to 1997.

Analysis of new treatment demand is open to a variety of confounding factors, many of which cannot be quantified. Changes in local heroin markets, onset to treatment lag, reporting compliance, treatment capacity, or services' attractiveness to clients, could each result in the observed increase in new treatment demands reported from Bolton. Our analysis indicates that onset to treatment lag may have declined in this area. As a result, the rate at which users emerged from the hidden population may have increased, hence the trend in new treatment demands may exaggerate the magnitude of an epidemic phase. However, the reduction in lag may, itself, indicate the emergence of a different population. Although difficult to quantify, changes in treatment provision warrant careful consideration. Treatment capacity increased in Bolton in around 1994 (personal communication), but this change post-dates the initial peak in new treatment demand (fig 1) and the onset of changes in age specific population rates (fig 4). Hence the increased provision is more likely to be a response to than a cause of the earlier increase in demand. Service capacity in Trafford has also increased during the period studied (personal communication).

Examination of onset trends between 1986 and 1992 produces results that are consistent with an increase in incidence of heroin use in Bolton (fig 2). Because of onset to treatment lag, some of those starting to use heroin in recent years have yet to appear in our treatment dataset. This proportion increases over time, hence trends for later years have not been examined here. As a result, this analysis does not enable us to comment on whether the apparent epidemic phase was sustained. However, its onset is consistent with the more sustained observed trends in treatment demand (fig 1) and age specific population rates (fig 4). Complex methods of correcting for the lag effect have been developed, ${ }^{10}$ and will enable examination of more recent onset trends.

Neither area has developed services targeted at young heroin users during the period
KEY POINTS

- There is geographical variation in the progression of heroin epidemics.

- The stage of an epidemic influences the composition and future size of the using population in the area affected.

- It is essential that governments' drug misuse strategies, and local responses to these, should consider underlying epidemic dynamics.

considered, hence it is very unlikely that changes in treatment provision underlie the observed trends in age specific population rates for Bolton (fig 4). The observed changes in the age composition of the treated population are highly consistent with an epidemic phase, characterised by an influx of younger users, and further reinforce our conclusions. Other work, involving prevalence estimation via multi-sample capture-recapture methods, confirms that younger users are over-represented among the population with drug problems in this area (unpublished data).

The dataset we have examined is the most readily available and detailed source of information about treated heroin use in the UK and is "what we have to work with". ${ }^{3}$ Clearly, no single analysis is a sufficient basis on which to draw firm conclusions concerning changes in incidence and a critical and cautious approach to findings is required. However, the analyses described here produce findings that, together, are highly consistent with differential trends in the two areas studied. We suggest that a heroin epidemic peaked before 1988 in Trafford; perhaps reducing the susceptible population through movement into, or "saturation" of the user population, ${ }^{4}$ or through "immunity" to starting use. Whatever the mechanism, the rate of new users entering the population was limited, resulting in a stable and older population. We suggest that the susceptible population was sufficiently large to feed the epidemic in Bolton between 1988 and 1995. Hence it is likely that the current population largely comprises younger users at an earlier stage of use.

On the basis of these observations, if incidence in Trafford is and remains stable, we predict that prevalence is likely to remain stable, as new users replace those leaving the population. However, in Bolton, because the bulk of the population seems to have started heroin use more recently, the population may continue to grow. Because each area starts from a different baseline position, each may experience different effects of intervention.

Results produced from crude application of the proposed performance indicators could be confounded by the underlying patterns of heroin use, leading to inaccurate perceptions of the efficacy of both current and future intervention strategies. For example, in Trafford, where the heroin epidemic seems at present to be contained, a reduction in the number of drug related deaths may reflect a decline in mortality rates. Whereas, in Bolton, although intervention may reduce rates for 
drug related mortality, a successful intervention is unlikely to be reflected in a reduction in the number of drug related deaths during a time frame that may encompass a dramatic growth in the prevalence of heroin use.

Targets must also be set within the context of possible future changes in prevalence. In the epidemic situation described in Bolton, the UK target to "increase the participation of problem drug users in drug treatment programmes by $100 \%$ by $2008^{\prime 11}$ may not keep pace with a growth in the prevalence of drug use locally. It is equally important for policy makers to consider how the future composition of their heroin using population should influence their intervention strategies. For example, the treatment needs of young people at an early stage of heroin use may require a different response to those of older and more established users.

In conclusion, our analyses provide a clear example of how areas may differ with respect to epidemic progression of heroin use. It is essential that governments' strategies, and local responses to these, should be cognisant of these dynamics.

Funding: ongoing funding for elements of this work has been provided by the Greater Manchester Drug Action Partnership (Single Regeneration Budget) and North West Health Authorities.

Conflicts of interest: none.
1 UK Government. Tackling drugs to build a better Britain: the government's ten year strategy for tackling drug misuse. London: UK Government, 1998.

2 De Alarcon R, Rathod NH. Prevalence and early detection of heroin abuse. BMF 1968;2:549-53.

3 Hunt LG, Chambers CD. The heroin epidemics. New York: Spectrum, 1976.

4 De Alarcon R. Lessons from a recent British Drug Outbreak. Conference proceedings of the London Royal Society of Medicine 1973;49-56.

5 Parker H, Newcombe R, Bakx K. The new heroin users: prevalence and characteristics in Wirral, Merseyside. BMF 1987;82:147-57.

6 Hamid A, Curtis R, McCoy K, et al. The heroin epidemic in New York City: current status and prognoses. $\mathcal{F}$ Psychoactive Drugs 1997;29:375-91.

7 Stimson GV, Hickman M, Quirk A, et al, eds. Estimating the prevalence of problem drug use in Europe. Luxemborg: European Monitoring Centre on Drugs \& Drug Addiction Scientific Monograph Series 1, Council of Europe/ EMCDDA, Office for Official Publications of the European Communities, 1997.

8 Stimson GV, Judd A. Estimating the scale and nature of drug problems: the relationship between science, policy and drugs strategy. In Stimson GV, et al, ed. Estimating the prevalence of problem drug use in Europe. Luxemborg: European Monitoring Centre on Drugs \& Drug Addiction Scientific Monograph Series 1, Council of Europe/ EMCDDA, Office for Official Publications of the European Communities, 1997:19-26.

9 Crabbe T, Donmall M, Millar T. Validation of the University of Manchester Drug Misuse Database. $\mathcal{F}$ Epidemiol Community Health 1999;53:159-64.

10 Hickman M, Seaman S, De Angelis D. Estimating the "relative" incidence of heroin use: an application of a method to adjust observed reports of presentations at specialist treatment agencies. Am F Epidemiol 2001;153:63241.

11 UK Government. The United Kingdom anti-drug coordinator's first annual report and national plan. London: Cabinet Office for the Central Office of Information, UK Government, May 1999 